\title{
Oxygenation impairment after total arch replacement with a stented elephant trunk for type-A dissection
}

\author{
Yuwen Shen, MD, Chuanzhen Liu, PhD, Changcun Fang, PhD, Jie Xi, MD, Shuming Wu, PhD,
} Xinyan Pang, PhD, and Guangmin Song, $\mathrm{PhD}$

\section{ABSTRACT}

Objective: To study the risk factors of oxygenation impairment in patients with type-A acute aortic dissection who underwent total arch replacement with a stented elephant trunk.

Methods: In this study, 169 consecutive patients were enrolled who were diagnosed with type-A acute aortic dissection and underwent a total arch replacement procedure at the Qilu Hospital of Shandong University between January 2015 and February 2017. Postoperative oxygenation impairment was defined as arterial oxygen partial pressure/inspired oxygen fraction $\leq 200$ with positive end expiratory pressure $\geq 5 \mathrm{~cm} \mathrm{H}_{2} \mathrm{O}$ that occurred within 72 hours of surgery. Perioperative clinical characteristics of all patients were collected and univariable analyses were performed. Risk factors associated with oxygenation impairment identified by univariable analyses were included in the multivariable regression analysis.

Results: The incidence of postoperative oxygenation impairment was $48.5 \%$. Postoperative oxygenation impairment was associated with prolonged mechanical ventilation time, intensive care unit stay, and hospital stay. Multivariable regression analysis demonstrated that body mass index (odds ratio [OR], 1.204; 95\% confidence interval [CI], 1.065-1.361; $P=.003)$, preoperative oxygenation impairment (OR, 9.768; 95\% CI, 4.159-22.941; $P<.001$ ), preoperative homocysteine (OR, 1.080; 95\% CI, 1.006-1.158; $P=.032$ ), circulatory arrest time (OR, $1.123 ; 95 \% \mathrm{CI}, 1.044-1.207 ; P=.002)$, and plasma transfusion (OR, 1.002; 95\% CI, 1.001-1.003; $P=.002$ ) were significantly associated with postoperative oxygenation impairment.

Conclusions: Postoperative oxygenation impairment is a common complication of surgery for type-A acute aortic dissection. Body mass index, preoperative oxygenation impairment, preoperative homocysteine, circulatory arrest time, and plasma transfusion were independent risk factors for oxygenation impairment after a total arch replacement procedure. (J Thorac Cardiovasc Surg 2018;155:2267-74)

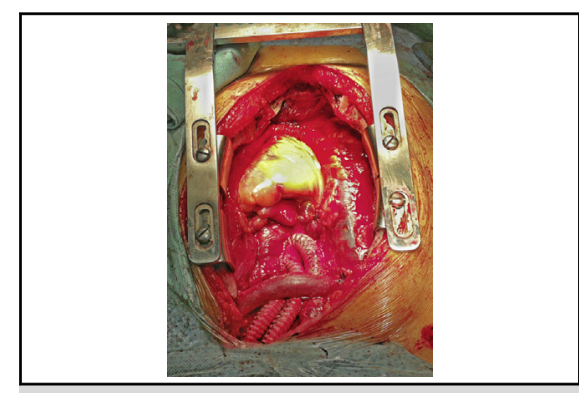

Total arch replacement procedure by a 4-branched graft.

\section{Central Message}

We studied risk factors of oxygenation impairment in patients after total arch replacement for acute type-A aortic dissection using multivariate regression analysis.

\section{Perspective}

Body mass index, preoperative hypoxemia, preoperative homocysteine, circulatory arrest time, and plasma transfusion were independent risk factors for oxygenation impairment of type- A aortic arch dissection after a total arch replacement procedure. Early discovery, mechanical ventilation strategy adjustment, inflammation control, transfusion and fluid control, and cardiac arrest time reduction all contribute to the improvement of clinical prognosis.

See Editorial Commentary page 2275.

See Editorial page 2251.

\footnotetext{
From the Department of Cardiovascular Surgery, Qilu Hospital of Shandong University, Shandong, China.

Supported by the Science Foundation of Qilu Hospital of Shandong (No. 2016QLQN20).

Drs Pang and Song contributed equally to this article.

Received for publication June 6, 2017; revisions received Jan 6, 2018; accepted for publication Jan 10, 2018; available ahead of print March 10, 2018.

Address for reprints: Guangmin Song, $\mathrm{PhD}$, Department of Cardiovascular Surgery, Qilu Hospital of Shandong University, Jinan 250012, Shandong, China (E-mail: shen51283@hotmail.com).

$0022-5223 / \$ 36.00$

Copyright (c) 2018 by The American Association for Thoracic Surgery

https://doi.org/10.1016/j.jtcvs.2018.01.085
}

Due to its high mortality and morbidity, aortic dissection is a challenging medical emergency. The International Registry of Acute Aortic Dissection report indicated that from 1996 to 2013, the percentage of patients with type-A

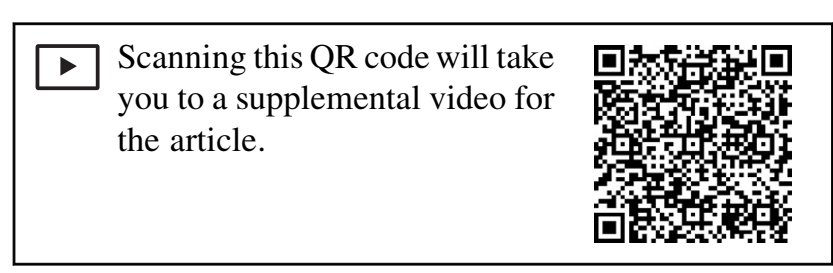




\section{Abbreviations and Acronyms}

$\mathrm{AAD}=$ acute aortic dissection

BMI = body mass index

$\mathrm{CA}=$ circulatory arrest

$\mathrm{CPB}=$ cardiopulmonary bypass

$\mathrm{FIO}_{2}=$ inspired oxygen fraction

ICU = intensive care unit

$\mathrm{PaO}_{2}=$ arterial oxygen partial pressure

PEEP $=$ positive end expiratory pressure

acute aortic dissection (AAD) that were referred to surgery increased from $78.7 \%$ to $90.2 \%$. Moreover, the surgical mortality rate decreased from $25.0 \%$ to $18 \%{ }^{1-4}$ Despite increased advances in diagnostic approaches, perioperative management, and surgical techniques, type-A AAD remains a major surgical challenge. The mortality associated with type-A AAD surgery remains extremely high. Patients often present with severe systemic pathophysiologic changes that may lead to multisystem and multiorgan perioperative complications, including respiratory dysfunction, renal dysfunction, and cerebral dysfunction. ${ }^{5}$ Respiratory dysfunction, as manifested by oxygenation impairment, is a common complication after type-A AAD surgery. ${ }^{6,7}$ Consequently, the duration of mechanical ventilation and intensive care unit (ICU) stay prolongs, thereby increasing the incidence of hospital-acquired pneumonia. Moreover, long-term serious deficient oxygen supply to multiple organs may lead to functional impairments, an increase in health care costs, and in the most severe cases, mortality. However, the mechanisms underlying oxygenation impairment of type-A AAD after total arch replacement are unclear and the number of studies that focus on this topic is limited. Therefore, it is of utmost importance to identify risk factors for oxygenation impairment after type-A AAD surgery to reduce perioperative complications and improve surgical treatment effects by early intervention and treatment of oxygenation impairment. In our study, we evaluated risk factors of postoperative oxygenation impairment in type-A AAD after total arch replacement.

\section{MATERIALS AND METHODS}

Consecutive patients who were diagnosed with type-A AAD (symptom onset within 2 weeks of surgery) and underwent total arch replacement at the Qilu Hospital of Shandong University, Jinan, China, were selected. The study was approved by the Ethics Committee of Qilu Hospital of Shandong University. Because of the retrospective nature of the study, any requirement for informed consent was waived. Given the high mortality rates in the acute phase, both drug and surgery treatments were started as quickly as possible when patients who were diagnosed with type-A AAD were admitted to our hospital. The diagnosis of type-A AAD was made using computed tomography angiography. Bedside chest radiographs were taken at 1,2 , and 3 days postoperatively. Pleural ultrasonography and echocardiography were conducted routinely at 1 day postoperation.
Echocardiography was undertaken to reassess left ventricular systolic and diastolic function if perioperative heart failure was suspected. Patients who experienced any perioperative complications that may influence oxygenation indices, such as cardiogenic pulmonary edema, atelectasis, pneumothorax, and large pleural effusion, were excluded from the study.

Surgeries were conducted by the same surgical team and involved a standard longitudinal median sternotomy. During surgery, the upper and lower limb blood pressures were measured through the left radial artery and the left foot dorsal artery. Moreover, myocardial protection was provided by intermittent, antegrade, cold blood cardioplegia solution via the right and left coronary ostia. The innominate artery, left carotid artery, and left subclavian artery were dissociated.

Cardiopulmonary bypass (CPB) was established by the right atrium and cannulation of the right axillary artery and femoral artery (the right or left femoral artery). After clamping the ascending aorta, the proximal end of the ascending aorta underwent aortic valvuloplasty or replacement and proximal graft anastomosis. The stent was implanted through the cut arch into the descending aorta. For total arch replacement surgery, a 4-branched graft was used to re-establish the aortic arch under hypothermic circulatory arrest (CA). First, anastomosis between the 4-branched graft trunk distal and the descending thoracic aorta was created. Subsequently, anastomosis between the aortic arch branches and the corresponding graft branches was made. Moreover, anastomosis between the graft trunk proximal aorta and the ascending aorta was performed. ${ }^{8,9}$ Before March 1, 2016, distal anastomosis was performed under deep hypothermic CA; that is, a nasopharyngeal temperature below $25^{\circ} \mathrm{C}$. However, after this date, distal anastomosis was performed using moderate hypothermic CA; that is, a nasopharyngeal temperature above $25^{\circ} \mathrm{C}$ and below $28^{\circ} \mathrm{C}$. To protect the brain during $\mathrm{CA}$, in addition to decreasing the temperature of the body, a patient's head is wrapped in ice bags and antegrade cerebral perfusion performed. Before January 2016, unilateral antegrade cerebral perfusion via the right axillary artery was performed. However, after January 2016,

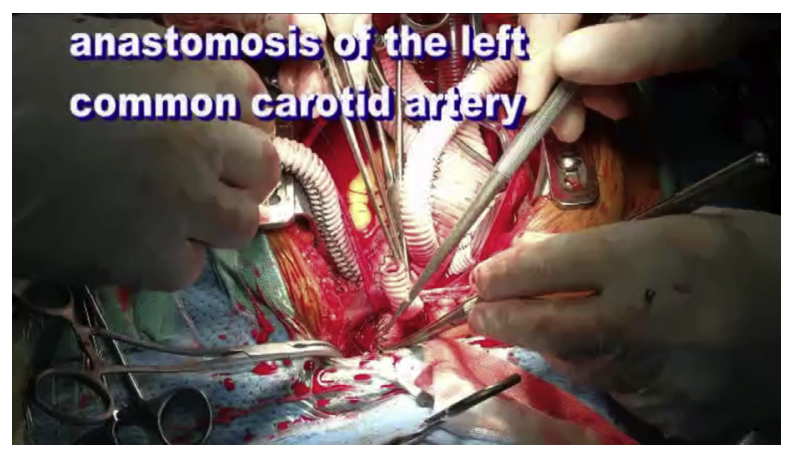

VIDEO 1. The procedure of total arch replacement with a stented elephant trunk underwent bilateral cerebral perfusion: dissociated right femoral artery, right axillary artery, innominate artery, left common carotid artery, and left subclavian artery. Cardiopulmonary bypass was established by cannulation of right atrium, right femoral artery, and right axillary artery. After clamping the ascending aorta, the proximal end of the ascending aorta was dealt with (aortic valvuloplasty and proximal graft anastomosis). When the patient was cooled to $26^{\circ} \mathrm{C}$ to $28^{\circ} \mathrm{C}$, bilateral antegrade cerebral perfusion via the right axillary artery and the left carotid artery was applied. The stent were implanted in the descending aorta through the cut arch, and then a total arch replacement procedure using a 4-branched graft to reestablish the aortic arch was performed. The sequence of anastomosis to the prosthetic graft was: left subclavian artery, left common carotid artery, proximal aortic stump, and innominate artery. Video available at: http:// www.jtcvsonline.org/article/S0022-5223(18)30366-0/fulltext. 
TABLE 1. Candidate variables for multivariable analyses selected by univariable analyses $(P<.05)$

\begin{tabular}{lr}
\hline \multicolumn{1}{c}{ Variable } & $\boldsymbol{P}$ value* \\
\hline Body mass index & $<.001$ \\
Preoperative oxygenation impairment & $<.001$ \\
\hline Preoperative homocysteine level & .028 \\
Preoperative white blood cell level & $<.001$ \\
\hline Preoperative creatinine level & .001 \\
cardiopulmonary bypass time & .033 \\
Circulatory arrest time & $<.001$ \\
\hline Last intraoperative central venous pressure & .001 \\
\hline Packed red blood cell transfusion & .012 \\
Plasma transfusion & $<.001$ \\
\hline Postoperative serum creatinine level & .001 \\
Postoperative nervous system dysfunction $\dagger$ & .003 \\
\hline *Based on univariable analyses. $\dagger$ Includes coma, cerebral infarction, paraplegia or \\
paraparesis, delirium, and other temporary neurologic dysfunctions after operation.
\end{tabular}

bilateral antegrade cerebral perfusion via the right axillary artery and the left carotid artery was applied. The sequence of anastomosis to the prosthetic graft in the unilateral cerebral perfusion group was as follows: descending aorta, left common carotid artery, proximal aortic stump, innominate artery, and left subclavian artery. In the bilateral cerebral perfusion group, the sequence was descending aorta, left subclavian artery, left common carotid artery, proximal aortic stump, and innominate artery (Video 1). ${ }^{10}$ During surgery, the Bentall procedure was performed in patients with severe aortic regurgitation and dilation of the aortic root. Patients presented with combined coronary artery disease underwent coronary artery bypass grafting, whereas patients who presented with mitral valve disease underwent mitral valve replacement.

Synchronized intermittent mandatory ventilation mode was used in all patients. Adjustment of the mechanical ventilation settings allowed for maintaining a correct lung gas exchange, including respiratory rate, tidal volume, inspired oxygen fraction $\left(\mathrm{FIO}_{2}\right)$, and using adequate values of positive end expiratory pressure (PEEP). When the arterial oxygen partial pressure to $\mathrm{FIO}_{2}$ ratio $\left(\mathrm{PaO}_{2}: \mathrm{FIO}_{2}\right)$ was poor, additional maneuvers were undertaken to optimize oxygenation. These included a low tidal volume ventilation, individualized setting of PEEP values, and decubitus changes (lateral and prone positions). The level of PEEP ranged from 5 to $15 \mathrm{~cm}$ $\mathrm{H}_{2} \mathrm{O}$ and was set according to the required level of $\mathrm{FIO}_{2}$. The patient's blood gas, PEEP levels, and $\mathrm{FIO}_{2}$ levels were measured and recorded preoperatively within 24 hours as well as postoperatively. The oxygenation index was calculated using $\mathrm{PaO}_{2}: \mathrm{FIO}_{2}$. Postoperative oxygenation impairment was defined as the oxygenation index $\leq 200$ with PEEP $\geq 5 \mathrm{~cm} \mathrm{H} \mathrm{H}_{2} \mathrm{O}$ within 72 hours of surgery (patients with a temporary, solvable conditions such as patient-ventilator conflict resulting in temporary hypo-oxygenation were reevaluated with another blood gas). ${ }^{11,12}$ Patients who were diagnosed with postoperative oxygenation impairment were assigned to the oxygenation impairment group; others were assigned to the nonoxygenation impairment group. As defined by the diagnostic criteria, 5 other end points were evaluated: time to extubation, length of ICU stay, length of hospital stay, hospital-acquired pneumonia, and death. ${ }^{13}$ Criteria to remove the extubation ventilator included that the patient was responsive and cooperative, $\mathrm{PaO}_{2} \geq 80 \mathrm{~mm}$ $\mathrm{Hg}$ and $\mathrm{FIO}_{2} \leq 0.4$, breathing rate $<30$ times/min, and a stable hemodynamic state. Hospital-acquired pneumonia developed at least 48 hours after hospital admission. Diagnosis was suspected on the basis of clinical presentation and chest radiograph and was confirmed by bronchoscopic sampling of the lower respiratory tract.
All data were retrospectively collected from medical records. The following perioperative parameters were evaluated and compared between the oxygenation impairment and nonoxygenation impairment groups: gender, age, body mass index (BMI), comorbidities, smoking history, preoperative blood cell counts, preoperative homocysteine level, preoperative complications, preoperative cardiac function, liver and renal function before and after surgery, time from symptoms onset to surgery, ${ }^{8} \mathrm{CPB}$ time, crossclamp time, CA time, minimal temperature, type of cerebral perfusion, surgical pattern, last intraoperative central venous pressure, perioperative packed red blood cells, plasma and platelets transfusions perioperatively (within 24 hours), and postoperative complications.

\section{Statistical Analysis}

Analyses were performed using IBM SPSS version 19.0 software (IBM-SPSS Inc, Armonk, NY). The Kolmogorov-Smirnov test was used to evaluate normality. Normally distributed continuous variables were expressed as the mean \pm standard deviation and were analyzed by $t$ test Nonnormally distributed data were expressed as the median (interquartile range) and compared using the Mann-Whitney $U$ test. Categorical variables were expressed as frequencies and analyzed by the $\chi^{2}$ test or Fisher exact test. Variables that were significantly associated with oxygenation impairment in univariable analyses $(P<.05)$ (Table 1) underwent multivariable binary logistic regression (backward and Akaike information criterion). Calibration of the model was assessed with the Hosmer and Lemeshow goodness-of-fit test.

\section{RESULTS}

A total of 176 patients were diagnosed with type-A AAD and underwent total arch replacement at the Qilu Hospital of Shandong University from January 2015 to February 2017. Of these 176 patients, 7 individuals $(1$ with atelectasis, 2 with pneumothorax, and 4 with large pleural effusion) were excluded. The 169 enrolled patients (age range, 25-72 years with average age, 52.21 years) included 121 men and 48 women. A total of 99 patients underwent ascending aorta, aortic arch replacement, and stent implantation. Moreover, 70 patients simultaneously underwent Bentall plus total arch replacement and stent implantation; 15 patients underwent ascending aorta, aortic arch replacement, and stent implantation combined with coronary artery bypass grafting; and 2 patients underwent ascending aorta, aortic arch replacement, and stent implantation combined with mitral valve replacement. The overall in-hospital mortality was $7.1 \%$ (12 out of 169 patients). The causes of death included viralactivated hepatitis type $\mathrm{B}$ that led to acute severe hepatic failure in 2 patients, a fungal infection in 1 patient, multiple organ dysfunction in 6 patients, myonephropathic metabolic syndrome in 1 patient, and a low cardiac output in 2 patients. No cases of intraoperative death were observed. In our study, the incidence of postoperative oxygenation impairment in the patient population was $48.5 \%(n=82)$. Ninety-five percent of patients $(n=78)$ with oxygenation impairment were identified within 12 hours of surgery, and the duration of oxygenation impairment ranged from 12 hours to more than 72 hours.

Among the preoperative characteristics, we found that BMI, preoperative serum creatinine levels, preoperative 
TABLE 2. The preoperative characteristics of patients

\begin{tabular}{|c|c|c|c|c|}
\hline Preoperative characteristic & All $(\mathbf{N}=169)$ & $\begin{array}{l}\text { Oxygenation impairment } \\
\text { group }(\mathbf{n}=\mathbf{8 2})\end{array}$ & $\begin{array}{l}\text { Nonoxygenation impairment } \\
\text { group }(\mathbf{n}=\mathbf{8 7})\end{array}$ & $P$ value \\
\hline Age $(y)$ & $51.21 \pm 9.735$ & $50.05 \pm 9.638$ & $52.31 \pm 9.754$ & .13 \\
\hline Male & $121(71.6)$ & $62(75.6)$ & $59(67.8)$ & .307 \\
\hline Female & $48(28.4)$ & $20(24.4)$ & $28(32.2)$ & \\
\hline Body mass index & $27(23.6-29.5)$ & $28.35(26.65-30.5)$ & $26(22.5-27.5)$ & $<.001$ \\
\hline Time from symptom onset to surgery (d) & $3(2-4.5)$ & $3(2-3)$ & $3(2-9)$ & .133 \\
\hline LVEF $<0.45$ & $7(4.1)$ & $2(2.4)$ & $5(5.7)$ & .445 \\
\hline Smoking history & $75(44.4)$ & $37(45.1)$ & $38(43.7)$ & .878 \\
\hline Hypertension & $148(87.6)$ & $75(91.5)$ & $73(84.0)$ & .165 \\
\hline Marfan syndrome & $10(5.9)$ & $3(3.7)$ & $7(8.0)$ & .331 \\
\hline Diabetes & $10(5.9)$ & $4(4.9)$ & $6(6.9)$ & .747 \\
\hline Coronary disease & $13(7.7)$ & $8(9.8)$ & $5(5.7)$ & .394 \\
\hline Chronic obstructive pulmonary disease & $1(0.6)$ & $1(1.2)$ & $0(0)$ & .485 \\
\hline Organ malperfusion* & $39(23.1)$ & $22(26.8)$ & $17(19.5)$ & .279 \\
\hline Nervous system & $6(3.6)$ & $3(3.7)$ & $3(3.4)$ & 1.0 \\
\hline Coronary artery & $4(2.4)$ & $1(1.2)$ & $3(3.4)$ & .621 \\
\hline Kidney & $19(11.2)$ & $10(12.2)$ & $9(10.3)$ & .809 \\
\hline Digestive system & $11(6.5)$ & $7(8.5)$ & $4(4.6)$ & .360 \\
\hline Limbs & $10(5.9)$ & $6(7.3)$ & $4(4.6)$ & .526 \\
\hline Severe aortic regurgitation & $68(40.2)$ & $29(35.4)$ & $39(44.8)$ & .27 \\
\hline Cardiac tamponade $\dagger$ & $17(10.1)$ & $7(8.5)$ & $10(11.5)$ & .613 \\
\hline $\mathrm{ALT}>40 \mathrm{u} / \mathrm{L}$ & $34(20.1)$ & $21(25.6)$ & $13(14.9)$ & .089 \\
\hline $\mathrm{AST}>80 \mathrm{u} / \mathrm{L}$ & $23(13.6)$ & $14(17.1)$ & $9(10.3)$ & .263 \\
\hline Preoperative creatinine $(\mu \mathrm{mol} / \mathrm{L})$ & $78(63-108.5)$ & $90(70-130.3)$ & $72(58-90)$ & .001 \\
\hline Preoperative homocysteine $(\mu \mathrm{mol} / \mathrm{L})$ & $13.9(11.2-18.5)$ & $15.25(11.6-19.73)$ & $13.3(11-16.8)$ & .028 \\
\hline Preoperative WBC count $\left(10^{3} / \mu \mathrm{L}\right)$ & $10.49(8.22-12.76)$ & $11.77(9.41-13.81)$ & $9.13(7.24-11.31)$ & $<.001$ \\
\hline Preoperative oxygenation impairment, $\mathrm{n}(\%) \ddagger$ & $71(42 \%)$ & $55(67.1 \%)$ & $16(18.4 \%)$ & $<.001$ \\
\hline
\end{tabular}

Values are presented as mean \pm standard deviation, $\mathrm{n}(\%)$, or median (interquartile range). $L V E F$, Left ventricular ejection fraction; $A L T$, alanine transaminase; $A S T$, glutamic oxaloacetic transaminase; $W B C$, white blood cell. *Organ malperfusion was defined as clinical manifestations or evidence of ischemia of 1 or more organs of heart, brain, spinal cord, kidney, liver, gastrointestinal tracts and limbs. †Intraoperatively confirmed effusion volume exceeding $500 \mathrm{~mL}$. $\ddagger$ Preoperative oxygenation impairment was defined as oxygenation index before surgery of 300 or lower.

homocysteine levels, preoperative white blood cell count, and preoperative oxygenation impairment $\left(\mathrm{PaO}_{2}: \mathrm{FIO}_{2} \leq 300\right)$ were significantly elevated in the oxygenation impairment group compared with the nonoxygenation impairment group $(P<.05)$. Age and gender were similar in the 2 groups (both $P$ values $>.05$ ). Moreover, comorbidities (eg, hypertension, diabetes, and coronary disease) and complications were similar in the 2 groups (all $P$ values $>.05$ ) (Table 2).

Among the intraoperative and postoperative characteristics, no significant differences were found in the incidence of oxygenation impairment based on the different types of surgery $(P>.05)$. CPB time, CA time, last intraoperative central venous pressure, transfusion of packed red blood cells and plasma within 24 hours, postoperative serum creatinine levels, and postoperative nervous system dysfunction were significantly increased in the oxygenation impairment group compared with the nonoxygenation impairment group (Table 3).

Multivariable regression analysis confirmed that BMI, preoperative oxygenation impairment $\left(\mathrm{PaO}_{2}: \mathrm{FIO}_{2} \leq 300\right)$, preoperative homocysteine level, CA time, and plasma transfusion were significant postoperative oxygenation impairment risk factors (Table 4). According to the Hosmer and Lemeshow goodness-of-fit test $P=.684>0.05$, the prediction model successfully predicted the incidence of postoperative oxygenation impairment.

Postoperative oxygenation impairment significantly associated with prolonged mechanical ventilation time, ICU stay, and hospital stay. Although postoperative oxygenation impairment was associated with higher incidence of hospital-acquired pneumonia, the rate of death was not increased (Table 5). 
TABLE 3. The intraoperative and postoperative characteristics of patients

\begin{tabular}{|c|c|c|c|c|}
\hline Characteristic & All $(N=169)$ & $\begin{array}{l}\text { Oxygenation impairment } \\
\text { group }(\mathbf{n}=\mathbf{8 2})\end{array}$ & $\begin{array}{c}\text { Nonoxygenation impairment } \\
\text { group }(n=87)\end{array}$ & $P$ value \\
\hline Concomitant Bentall* & $70(41.4)$ & $30(36.6)$ & $40(46)$ & .215 \\
\hline Concomitant CABG & 15 (41.4) & $8(9.8)$ & $7(8.0)$ & 696 \\
\hline Concomitant MVR & $2(41.4)$ & $0(0)$ & $2(2.3)$ & .497 \\
\hline CPB time (min) & $234(210-266)$ & $241(214.5-273)$ & $230(209-256)$ & .033 \\
\hline Crossclamp time (min) & $156(135-175)$ & $160.5(141.75-181)$ & $152(131-170)$ & .066 \\
\hline Circulatory arrest time (min) & $25(23-29)$ & $27(24-30)$ & $24(22-27)$ & $<.001$ \\
\hline Nasopharyngeal temperature $\left({ }^{\circ} \mathrm{C}\right)$ & $25(25-28)$ & $25(25-28)$ & $28(25-28)$ & 164 \\
\hline Bilateral antegrade cerebral perfusion & $97(57.4)$ & $49(59.8)$ & $48(55.2)$ & .641 \\
\hline Last intraoperative CVP (mm Hg) & $12(9-14)$ & $12(10-14.25)$ & $11(8-13)$ & .001 \\
\hline PRBC transfusion $(\mathrm{u})$ & $6(4-8)$ & $6.5(4-8)$ & $6(4-8)$ & .012 \\
\hline Plasma transfusion $(\mathrm{mL})$ & $800(600-1100)$ & $950(800-1300)$ & $750(600-900)$ & $<.001$ \\
\hline Platelet transfusion $(\mathrm{U})$ & $2(1,2)$ & $2(1,2)$ & $2(1,2)$ & .131 \\
\hline Postoperative ALT > $40 \mathrm{IU} / \mathrm{L}$ & $58(34.3)$ & $34(41.5)$ & $24(27.6)$ & .74 \\
\hline Postoperative AST > $80 \mathrm{IU} / \mathrm{L}$ & 70 (41.4) & $38(46.3)$ & $32(36.8)$ & .207 \\
\hline Postoperative serum creatinine $(\mu \mathrm{mol} / \mathrm{L})$ & $106(74-156)$ & $123(84.25-187)$ & $91(70-121)$ & .001 \\
\hline Dialysis & $10(5.9)$ & $8(9.8)$ & $2(2.3)$ & .052 \\
\hline Postoperative nervous system dysfunction $\dagger$ & $75(44.4)$ & $46(56.1)$ & $29(33.3)$ & .003 \\
\hline
\end{tabular}

Values are presented as $\mathrm{n}(\%)$ or median (interquartile range). $C A B G$, Coronary artery bypass grafting; $M V R$, mitral valve replacement; $C P B$, cardiopulmonary bypass; $C V P$, central venous pressure; $P R B C$, packed red blood cell; $A L T$, alanine transaminase; $A S T$, glutamic oxaloacetic transaminase. *Aortic valve replacement with a valved conduit. $\dagger$ Includes coma, cerebral infarction, paraplegia or paraparesis, delirium, and other temporary neurologic dysfunctions after operation.

\section{DISCUSSION}

Acute respiratory distress syndrome is a leading cause of postoperative oxygenation impairment. ${ }^{14}$ According to both the Berlin and the American European Consensus Conference, a criterion of acute respiratory distress syndrome diagnosis is degree of oxygenation impairment. ${ }^{12,13}$ Furthermore, due to the application of sternotomy and CPB for surgery to address type-A AAD, it was difficult to use typical bilateral opacities radiographic images as a diagnostic criterion. Therefore, in the present study, we used oxygenation impairment as the single diagnostic criterion for ARDS and postoperative oxygenation impairment was defined as $\mathrm{PaO}_{2}: \mathrm{FIO}_{2}$ $\leq 200 \mathrm{~mm} \mathrm{Hg}$ with PEEP $\geq 5 \mathrm{~cm} \mathrm{H}_{2} \mathrm{O}$ within 72 hours of surgery. ${ }^{12,13}$ Postoperative oxygenation impairment had an adverse effect on the duration of mechanical ventilation time, the incidence of hospital-acquired pneumonia, and the length of ICU and hospital stay.
Determining risk factors that predispose postoperative oxygenation impairment are essential for improving a patient's clinical course and reducing medical costs. Our aim was to identify the incidence and risk factors of oxygenation impairment after total arch replacement for type-A AAD to provide clinically relevant information to better manage patients' health. In the current study, the incidence of postoperative oxygenation impairment was $48.5 \%$. Parameters, including BMI, preoperative oxygenation impairment, preoperative homocysteine level, CA time, and plasma transfusion were identified as risk factors.

Obesity is known as a risk factor for postoperative oxygenation impairment. ${ }^{6,9}$ Aizawa and colleagues ${ }^{15}$ reported that the incidence of postoperative oxygenation impairment in type-A AAD was increased in patients with BMI $\geq 25$ compared with patients with BMI $<25$ $(81.8 \%$ vs $53.6 \% ; P=.036)$. This is in line with the

TABLE 4. Risk factors for oxygenation impairment in the multivariable backward regression analyses

\begin{tabular}{lcccc}
\hline \multicolumn{1}{c}{ Risk factor } & Coefficient & Odds ratio & 95\% confidence interval & $P$ value \\
\hline Body mass index & 0.186 & 1.204 & $1.065-1.361$ & .003 \\
Preoperative hypoxemia & 2.279 & 9.768 & $4.159-22.941$ & $<.001$ \\
Preoperative homocysteine level & 0.077 & 1.080 & $1.006-1.158$ & .032 \\
Circulatory arrest time & 0.116 & 1.123 & $1.044-1.207$ & .002 \\
Plasma transfusion & 0.002 & 1.002 & $1.001-1.003$ & .002 \\
\hline
\end{tabular}


TABLE 5. Univariable analyses of the postoperative conditions

\begin{tabular}{|c|c|c|c|c|}
\hline Postoperative condition & All (N = 169) & $\begin{array}{l}\text { Oxygenation impairment } \\
\text { group }(\mathbf{n}=\mathbf{8 2})\end{array}$ & $\begin{array}{l}\text { Nonoxygenation impairment } \\
\text { group }(\mathbf{n}=\mathbf{8 7})\end{array}$ & $P$ value \\
\hline Mechanical ventilation time (h) & $33.5(15.25-62)$ & $59(34-88.125)$ & $17.5(13.5-34)$ & $<.001$ \\
\hline Intensive care unit stay (h) & $126(97-158.5)$ & $153.5(129.25-205)$ & $103(79.5-125)$ & $<.001$ \\
\hline Hospital stay (d) & $18(16-21)$ & $20(16.75-24)$ & $17(15-19)$ & $<.001$ \\
\hline Hospital-acquired pneumonia & $13(7.7)$ & $10(12.2)$ & $3(3.4)$ & .043 \\
\hline Death & $12(7.1)$ & $7(8.5)$ & $5(5.7)$ & .480 \\
\hline
\end{tabular}

Values are presented as median (interquartile range) or $\mathrm{n}(\%)$.

findings in our study, in which we demonstrated that BMI was a risk factor for oxygenation impairment after total arch replacement. Previous studies have showed that obesity and oxygenation impairment are involved in different mechanisms. Obesity, for example, affects the physiology of the respiratory system. Moreover, the combination of impaired respiratory system compliance, mismatched ventilation/perfusion distribution, excess respiration load, and a reduced ventilatory drive predispose obese individuals to postoperative oxygenation impairment. $^{16,17}$

Studies have shown that preoperative oxygenation impairment is common among patients with type-A AAD and significantly increased postoperative oxygenation impairment incidence. ${ }^{6,7}$ In our study, we found that the incidence of preoperative oxygenation impairment was $42 \%$. It is well accepted that at the onset of AAD, systemic inflammatory reactions are initiated. Indeed, Hasegawa and colleagues ${ }^{18}$ reported that oxygenation was impaired from the onset of dissection in patients who were conservatively treated for $\mathrm{AAD}$, and that interleukin-8 levels were elevated in patients with impaired oxygenation. The inflammatory cascade releases proinflammatory cytokines, leading to neutrophil accumulation and activation. Subsequently, activated neutrophils release toxic mediators and proteolytic enzymes that may injure alveolar capillaries and alveolar epithelial cells. ${ }^{11}$ Oxygenation was impaired by the inflammatory cascade, and preoperative oxygenation impairment occurred. Moreover, previous reports have indicated that a preoperative inflammatory state severely influenced the intra- and postoperative degree of systemic inflammation. ${ }^{6}$ Therefore, postoperative oxygenation impairment is related to preoperative impairment in oxygenation, which is consistent with the findings presented in our study.

Disruption of homocysteine metabolism causes hyperhomocysteinemia, a condition that is associated with the impairment of nitric oxide bioavailability, tissue hypoxia, and an increased risk of vascular disease. In this study, we found that hyperhomocysteinemia was related to postoperative oxygenation impairment. The mechanism may be associated with impaired endothelial cells, oxidative stress, and coagulation abnormalities. Studies report that homocysteine impairs endothelial cells, attenuates antithrombotic effects of the endothelium, and inactivates nitric oxide via oxygen radicals resulting in apoptosis, thrombosis, and platelet activation. ${ }^{19}$ This process impaired oxygenation function and led to hypoxemia.

Postoperative oxygenation impairment can often be detected in patients who undergo cardiac surgery with CPB. ${ }^{20,21}$ Significant improvements were made in the CPB approach; however, $20 \%$ to $25 \%$ of patients still experience various degrees of lung dysfunction after cardiac surgery. ${ }^{22,23} \mathrm{CPB}$ and $\mathrm{CA}$ have been reported to play important roles in postoperative oxygenation impairment. ${ }^{6,7,9} \mathrm{CPB}$ represents a type of nonphysiologic circulation that influences peripheral tissue perfusion. However, in the event of CA, tissues are either hypoperfused or not perfused at all, leading to extensive ischemia-reperfusion injuries. ${ }^{9}$ An important approach for type-A AAD surgery is deep hypothermia. Deep hypothermia can slow down the cellular metabolism and reduce cellular damage during CA. However, hypothermia also influences the coagulation system and the activity of enzymes and platelets, which can cause hemorrhage and may require massive blood transfusion ${ }^{24}$ that may result in transfusion-related acute lung injury. Furthermore, microthrombosis, cellular debris, foreign proteins, and inflammatory mediators can directly influence oxygenation function. Prolonged hypoperfusion, ischemia-reperfusion injuries, hypothermia, and blood transfusion may result in the release of activated oxygen species and inflammatory molecules into the circulation. This may activate WBCs, platelets, complements, and the blood coagulation system. An uncontrolled inflammatory cascade may lead to increased permeability of capillary membranes, hypoperfusion, and tissue anoxia, thereby causing multiorgan dysfunction, including respiratory dysfunction. ${ }^{25-28}$ In addition, it was found that CA time and plasma transfusion were associated with postoperative oxygenation impairment.

The process of postoperative oxygenation impairment is a combination of many factors in which the inflammatory response plays an important role. Based on our findings, identifying and adjusting risk factors early, administering perioperative anti-inflammatory treatment, reducing blood 
transfusion (ie, rapid and effective bleeding control, preoperative autologous platelet collection, and intraoperative cell salvage), and shortening the time of CA should help decrease the incidence of postoperative oxygenation impairment. Treacher and colleagues $^{29}$ reported that leucofiltration safely and effectively removes circulating polymorphonuclear cells from patients with systemic inflammatory response syndrome. This may result in improved pulmonary function in these patients. De Santo and colleagues ${ }^{30}$ reported that pulmonary perfusion during hypothermic CA preserved increased lung function. For patients at a high risk of postoperative oxygenation impairment, leucofiltration and pulmonary perfusion may be considered. However, additional studies will be required to verify this statement.

Although promising, our study has some limitations. First, $\mathrm{PaO}_{2}$ is dependent on $\mathrm{FIO}_{2}$, so we adopted $\mathrm{PaO}_{2}: \mathrm{FIO}_{2}$ instead of $\mathrm{PaO}_{2}$ to evaluate oxygenation. But $\mathrm{PaO}_{2}: \mathrm{FIO}_{2}$ still could be influenced by factors that are difficult to control, including the hemoglobin dissociation curve, difference in oxygen extraction, and manipulation by PEEP. Second, because of the retrospective nature of our study, the results may have been influenced by the nonrandomized nature. Some data, including the history of chronic pulmonary disease and the compliance of lungs, were inaccurate. Third, our study was a single-center study in which all patients were from the Department of Cardiac Surgery at Qilu Hospital, Jinan, China, and the sample size was limited. Moreover, patients did not receive follow-up after discharge; therefore, long-term survival remains unknown.

\section{CONCLUSIONS}

The data presented in this study indicate that for patients with type-A AAD, postoperative oxygenation impairment after total arch replacement is common and associated with prolonged ventilation time, extended ICU and hospital stays, and a higher incidence of hospital-acquired pneumonia. BMI, preoperative oxygenation impairment, preoperative homocysteine level, CA time, and plasma transfusion were independently associated with postoperative oxygenation impairment in patients with type-A AAD. Early discovery, early diagnosis, early treatment of oxygenation impairment, controlling inflammatory reactions, reducing intra- and postoperative plasma transfusion, and shortening of the CA time may contribute to improving the postoperative clinical course of patients undergoing total arch replacement surgery for type-A AAD.

\section{Conflict of Interest Statement}

Authors have nothing to disclose with regard to commercial support.

\section{References}

1. Chiu P, Miller DC. Evolution of surgical therapy for Stanford acute type-A aortic dissection. Ann Cardiothorac Surg. 2016;5:275-95.

2. Mody PS, Wang Y, Geirsson A, Kim N, Desai MM, Gupta A, et al. Trends in aortic dissection hospitalizations, interventions, and outcomes among medicare beneficiaries in the United States 2000-2011. Circ Cardiovasc Qual Outcomes 2014;7:920-8

3. Pape LA, Awais M, Woznicki EM, Suzuki T, Trimarchi S, Evangelista A, et al. Presentation, diagnosis, and outcomes of acute aortic dissection: 17-year trends from the international registry of acute aortic dissection. J Am Coll Cardiol. 2015;66:350-8.

4. Fukunaga N, Koyama T. Evolution of diagnosis and clinical outcomes in acute aortic dissection: data from the international registry of acute aortic dissection. J Thorac Dis. 2016;8:E625-7.

5. Wong DR, Lemaire SA, Coselli JS. Managing dissections of the thoracic aorta. Am Surg. 2008;74:364-80.

6. Nakajima T, Kawazoe K, Izumoto H, Kataoka T, Niinuma H, Shirahashi N. Risk factors for hypoxemia after surgery for acute type A aortic dissection. Surg Today. 2006;36:680-5.

7. Liu N, Zhang W, Ma W, Shang W, Zheng J, Sun L. Risk factors for hypoxemia following surgical repair of acute type A aortic dissection. Interact Cardiovasc Thorac Surg. 2017;24:251-6.

8. Sun LZ, Ma WG, Zhu JM, Zheng J, Liu YM, Ziganshin BA, et al. Sun's procedure for chronic type A aortic dissection: total arch replacement using a tetrafurcate graft with stented elephant trunk implantation. Ann Cardiothorac Surg. 2013;2:665-6.

9. Wang Y, Xue S, Zhu H. Risk factors for postoperative hypoxemia in patients undergoing Stanford A aortic dissection surgery. J Cardiothorac Surg. 2013;8:118.

10. Tong G, Zhang B, Zhou X, Tao Y, Yan T, Wang X, et al. Bilateral versus unilateral antegrade cerebral perfusion in total arch replacement for type A aortic dissection. J Thorac Cardiovasc Surg. 2017; 154:767-75.

11. Bernard GR, Artigas A, Brigham KL, Carlet J, Falke K, Hudson L, et al. The American-European consensus conference on ARDS. Definitions, mechanisms, relevant outcomes, and clinical trial coordination. Am J Respir Crit Care Med. 1994;149:818-24.

12. ARDS Definition Task Force, Ranieri VM, Rubenfeld GD, Thompson BT, Ferguson ND, Caldwell E, Fan E, et al. Acute respiratory distress syndrome: the Berlin definition. JAMA. 2012;307:2526-33.

13. American Thoracic Society. Infectious Diseases Society of America guidelines for the management of adults with hospital-acquired, ventilator-associated, and healthcare-associated pneumonia. Am J Respir Crit Care Med. 2005;171:388-416.

14. Filsoufi F, Rahmanian PB, Castillo JG, Chikwe J, Adams DH. Predictors and early and late outcomes of respiratory failure in contemporary cardiac surgery. Chest. 2008;133:713-21.

15. Aizawa K, Sakano Y, Ohki S, Saito T, Konishi H, Misawa Y. Obesity is a risk factor of young onset of acute aortic dissection and postoperative hypoxemia. Japan J Thorac Surg. 2013;66:437-44.

16. Ranucci M, Ballotta A, La Rovere MT, Castelvecchio S. Postoperative hypoxemia and length of intensive care unit stay after cardiac surgery: the underweight paradox. PLoS One. 2014;9:e93992.

17. Ray CS, Sue DY, Bray G, Hansen JE, Wasserman K. Effects of obesity on respiratory function. Am Rev Respir Dis. 1983;128:501-6.

18. Hasegawa Y, Ishikawa S, Ohtaki A, Otani Y, Takahashi T, Sato Y, et al. Impaired lung oxygenation in acute aortic dissection. J Cardiovasc Surg (Torino). 1999;40:191-5.

19. Sawle P, Foresti R, Green CJ, Motterlini R. Homocysteine attenuates endothelial haem oxygenase-1 induction by nitric oxide (NO) and hypoxia. FEBS Lett. 2001; 508:403-6.

20. Filsoufi F, Rahmanian PB, Castillo JG, Chikwe J, Adams DH. Logistic risk mode predicting postoperative respiratory failure in patients undergoing valve surgery. Eur J Cardiothorac Surg. 2008;34:953-9.

21. Ji Q, Mei Y, Wang X, Feng J, Cai J, Sun Y, et al. Study on the risk factors of postoperative hypoxemia in patients undergoing coronary artery bypass grafting. Circ J. 2008;72:1975-80.

22. Apostolakis E, Filos KS, Koletsis E, Dougenis D. Lung dysfunction following cardiopulmonary bypass. J Card Surg. 2010;25:47-55.

23. Stephens RS, Shah AS, Whitman GJ. Lung injury and acute respiratory distress syndrome after cardiac surgery. Ann Thorac Surg. 2013;95:1122-9.

24. Algarni KD, Yanagawa B, Rao V, Yau TM. Profound hypothermia compared with moderate hypothermia in repair of acute type A aortic dissection. $J$ Thorac Cardiovasc Surg. 2014;148:2894-5. 
25. Roumen RM, Hendriks T, van der Ven-Jongekrijg J, Nieuwenhuijzen GA, Sauerwein RW, van der Meer JW, et al. Cytokine patterns in patients after major vascular surgery, hemorrhagic shock, and severe blunt trauma. Relation with subsequent adult respiratory distress syndrome and multiple organ failure. Ann Surg. 1993;218:769-76.

26. Asimakopoulos G. Systemic inflammation and cardiac surgery: an update. Perfusion. 2001;16:353-60.

27. Rothenburger M, Soeparwata R, Deng MC, Schmid C, Berendes E, Tjan TD, et al. Prediction of clinical outcome after cardiac surgery: the role of cytokines, endotoxin and anti-endotoxin core antibodies. Shock. 2001;16(Suppl I):44-50.

28. Székely A, Cserép Z, Sápi E, Breuer T, Nagy CA, Vargha P, et al. Risks and predictors of blood transfusion in pediatric patients undergoing open heart operations. Ann Thorac Surg. 2009;87:187-97.
29. Treacher DF, Sabbato M, Brown KA. The effects of leucodepletion in patients who develop the systemic inflammatory response syndrome following cardiopulmonary bypass. Perfusion. 2001;16(Suppl):67-73.

30. De Santo LS, Romano G, Amarelli C, Cotrufo M. Surgical repair of acute type A aortic dissection: continuous pulmonary perfusion during retrograde cerebral perfusion prevents lung injury in a pilot study. J Thorac Cardiovasc Surg. $2003 ; 126: 826-31$.

Key Words: acute aortic dissection, total arch replacement with a stented elephant trunk, oxygenation impairment, acute respiratory distress syndrome, oxygenation index

Readers who found these articles interesting may also like to read the following papers found in recent and future issues of our sister publications, Seminars in Thoracic and Cardiovascular Surgery and Operative Techniques in Thoracic and Cardiovascular Surgery!

\section{Adult: Aorta}

ORIGINAL SUBMISSION: Best Medical Treatment and Selective Stent-Graft Repair for Acute Type B Aortic Intramural Hematoma. Gabriele Piffaretti. Semin Thoracic Surg 2017: In press.

ORIGINAL SUBMISSION: Fluctuations in Spinal Cord Perfusion Pressure: a Harbinger of Delayed Paraplegia after Thoracoabdominal Aortic Repair. Harleen K. Sandhu. Semin Thoracic Surg 2017: 451-459.

Editorial Commentary: Keep Alert Eyes on Delayed Paraplegia. Kenji Minatoya. Semin Thoracic Surg 2017: 460-461.

Editorial Commentary: Problem Delayed Does Not Mean Solution Denied. Joseph S. Coselli. Semin Thoracic Surg 2017: 462-463. ORIGINAL SUBMISSION: Awake Thoracic Endovascular Aneurysm Repair for Aortic Rupture: A Case Series. Jessica Forcillo. Semin Thoracic Surg 2017: In press.

Editorial Commentary: Awake TEVAR for Ruptured Thoracic Aneurysms: Less is More? Akiko Tanaka. Semin Thoracic Surg 2017 In press.

ORIGINAL SUBMISSION: Impact of Discordant Views in the Management of Descending Thoracic Aortic Aneurysm. Peter Chiu. Semin Thoracic Surg 2017: 283-291.

Editorial Commentary: Surgery Is in the Eye of the Beholder. Anthony L. Estrera. Semin Thoracic Surg 2017: $292-293$. 